\title{
Integrated analysis of microfibrillar-associated proteins reveals MFAP4 as a novel biomarker in human cancers
}

\author{
Jue Yang ${ }^{\ddagger 1,1,2}$, Hui Song ${ }^{\ddagger, 3,4}$, Li Chen ${ }^{5}$, Kun CaO ${ }^{6}$, Yongqiang Zhang ${ }^{7}$, Yanmei $\mathrm{Li}^{\star, 1,2}$ \& \\ Xiaojiang $\mathrm{HaO}^{* *, 1,2}$ \\ ${ }^{1}$ The State Key Laboratory of Functions \& Applications of Medicinal Plants, Guizhou Medical University, Guiyang 550014, PR China \\ ${ }^{2}$ The Key Laboratory of Chemistry for Natural Products of Guizhou Province \& Chinese Academic of Sciences, Guiyang 550014, PR \\ China \\ ${ }^{3}$ The Key Laboratory of Endemic \& Ethnic Diseases, Guizhou Medical University, Ministry of Education, Guiyang 550004, PR China \\ ${ }^{4}$ The Key Laboratory of Medical Molecular Biology, Guizhou Medical University, Guizhou Province, Guiyang 550004, PR China \\ ${ }^{5}$ Guiyang University of Chinese Medicine, School of Pharmaceutical Sciences, Guiyang 550025, PR China \\ ${ }^{6}$ Department of General Surgery, Affiliated Hospital of Guizhou Medical University, Guiyang 550001, PR China \\ ${ }^{7}$ Guizhou University, School of Pharmaceutical Sciences, Guiyang, 550025, PR China \\ *Author for correspondence: liyanmei518@hotmail.com \\ **Author for correspondence: haoxj2013@126.com \\ $\ddagger$ Authors contributed equally
}

\begin{abstract}
Aim: The potential functions and underlying mechanism of microfibrillar-associated proteins (MFAPS) are explored in human cancers. Materials \& methods: Here, we examined the expression profiles, prognostic values, epigenetic and genetic alterations of MFAPs in human cancers from public omics repository. Results: Among MFAPs family, MFAP4 was frequently downregulated in the most human cancers and high mRNA expression of MFAP4 significantly correlated with better overall survival in breast cancer. DNA hypermethylation in the promoter of MFAP4 decreased its MRNA expression. MFAP4 strongly associated with pathway in impairment and alteration of the elastic fibers. Conclusion: This integrated analysis provides new insights into MFAPs in human cancers and indicates that MFAP4 could be used as novel biomarker for developing therapies against human cancers.
\end{abstract}

First draft submitted: 4 June 2018; Accepted for publication: 23 July 2018; Published online:

9 August 2018

Keywords: breast cancer $\bullet$ DNA methylation $\bullet$ microfibrillar-associated protein $4 \bullet$ overall survival $\bullet$ TCGA

Cancer is a highly complex trait that threatens billions of people worldwide. It is now clear that cancer is a consequence of the interaction of multiple acquired genetic and epigenetic lesions influenced by heredity and environment. Cancer cells can survive and proliferate under extremely adverse microenvironment, induce and maintain angiogenesis, migrate and invade into the surrounding tissues. Any molecule contributing to these processes is worthy of particular interest.

Accumulated studies demonstrate that microfibrils provide structural integrity of specific organ systems, but they can also provide a scaffold for elastogenesis in elastic tissues [1]. Interestingly, an elastin plays a role in cell adhesion, cell migration, survival and differentiation and has the ability to participate in cell signaling $[2,3]$. The microfibrillar-associated proteins (MFAPs) are located to microfibrils and can interact with fibrillin to influence microfibril function. Those evidence indicate $M F A P$ s may play an important role in the phenotype of cancer cells. So far, five MFAPs family members have been identified, MFAP1, MFAP2, MFAP3, MFAP4 and MFAP5. MFAP2 and MFAP5 encode protein MAGP-1 and MAGP-2, respectively. MFAP1, MFAP3 and MFAP4 cannot encode MAGP proteins but they were grouped together with MAGPs due to their small size and localization to microfibrils. Among them, MFAP2, MFAP4 and MFAP5 encode extracellular matrix proteins that play important biological roles in multiple organs, including bone, blood vessels, hemostasis and the immune system $[4,5]$. Recent 
findings show that MFAP1 associates with spliceosome function and is required for pre-mRNA processing [6-8]. MFAP3 functions as a nuclear kinase [9]. However, the prognostic significance, potential functions and molecular mechanism of MFAPs in tumorigenesis and tumor development remains largely unknown.

With the fast development and reduced costs of high throughput technologies, extensive public efforts to annotate of cancer genomes and seek for susceptibility loci facilitate researchers to illuminate molecular mechanism of human tumors as well as develop new strategies for precision therapy. For example, the Cancer Genome Atlas (TCGA) project has generated comprehensive, multidimensional maps of the key genomic changes in 33 types of cancer. By profiling gene expression, epigenetic modifications, copy-number variation and somatic mutations of more than 11,000 patients, this project accelerates the understanding of the molecular basis of cancer and improves our ability to diagnose, treat and prevent human cancers.

In this study, we performed a deep analysis of gene mRNA expression, genetic mutation and methylation modifications of MFAPs in breast carcinoma and other human cancers based on TCGA data. We also explored the potential prognostic values and gene regulatory networks of MFAPs involved in carcinogenesis and cancer progression.

\section{Materials \& methods}

\section{MFAPs-mRNA expression analysis}

MFAPs-mRNA expressions in different human cancers were analyzed using Oncomine gene expression array datasets [10]. The cut-off of p-value and fold change were defined as 0.01 and 2 , respectively. UALCAN is a web portal to perform in-depth analyses of gene expression in various tumor subgroups based on individual clinicopathologic features from the TCGA data. MFAPs-mRNA expression level in different cancer subtypes and grades/stages was evaluated using UALCAN [11].

\section{Kaplan-Meier survival analysis}

The correlation between the MFAPs expression and prognosis was analyzed using Kaplan-Meier plotter (KM plotter) [12]. We chose 'overall survival' (OS), 'auto-select best cut-off' and 'only JetSet best probe set' to calculate and plot Kaplan-Meier survival curves. Best cut-off values were generated through computing all possible cut-off values between the lower and upper quartiles and the best performing threshold was used as a cut-off (Supplementary Table 1). Statistical significance was determined by log-rank p-value and hazard ratios (HRs) with $95 \%$ CIs are displayed. The Affymetrix probeset IDs of MFAPs in breast, lung and stomach cancers are shown as follows: MFAP1: 203406_at; MFAP2: 203417_at; MFAP3: 213123_at; MFAP4: 212713_at and MFAP5: 213764_at. RNA-Seq ID of MFAP4 in liver cancer is 4239. GSE62254 datasets were excluded when KM plots were generated for MFAP4 in stomach cancer because GSE62254 has markedly different characteristics (longer survivals, shifted expression) than the other datasets.

\section{Mutations \& copy-number alterations analysis}

The cBioPortal (www.cbioportal.org) is an open access resource for interactive exploration of multidimensional cancer genomics datasets [13]. Breast invasive carcinoma (TCGA, Provisional, 1105 samples) was selected to analyze mutations and copy-number alterations of MFAP4. GISTIC 2.0 were used to identify significant somatic copynumber alterations, such as amplifications and deletions [14].

\section{Methylation modifications analysis}

To plot the relationship between DNA methylation and MFAP4 mRNA expression, 871 breast invasive carcinoma samples in MEXPRESS datasets were analyzed [15]. Then we used MethHC which included 839 breast invasive carcinoma samples to check the correlation of DNA methylation in the promoter and MFAP4-mRNA expression [16].

\section{Gene regulatory networks analysis}

Functional protein association network for MFAP4 was constructed using the Search Tool for the Retrieval of Interacting Genes/Proteins (STRING) with a confidence score of 0.4 [17]. Metascape was used for gene ontology and pathway enrichment analysis of the MFAP4-related genes [18]. 


\begin{tabular}{|r|}
\hline Analysis type by cancer \\
\hline Bladder cancer \\
\hline Brain and CNS cancer \\
\hline Breast cancer \\
\hline Cervical cancer \\
\hline Colorectal cancer \\
\hline Esophageal cancer \\
\hline Gastric cancer \\
\hline Head and neck cancer \\
\hline Kidney cancer \\
\hline Leukemia \\
\hline Liver cancer \\
\hline Lung cancer \\
\hline Lymphoma \\
\hline Melanoma \\
\hline Myeloma \\
\hline Other cancer \\
\hline Ovarian cancer \\
\hline Pancreatic cancer \\
\hline Prostate cancer \\
\hline Sarcoma \\
\hline Significant unique analyses \\
\hline unique analyses \\
\hline
\end{tabular}

\begin{tabular}{|c|c|c|c|c|c|c|c|c|c|}
\hline \multicolumn{7}{|c|}{ Cancer vs normal } \\
\hline MFAP1 & \multicolumn{2}{|c|}{ MFAP2 } & \multicolumn{2}{|c|}{ MFAP3 } & \multicolumn{2}{|c|}{ MFAP4 } & \multicolumn{2}{|c|}{ MFAP5 } \\
\hline & & 2 & & & & & 7 & & 3 \\
\hline 1 & & 1 & & 1 & 1 & 2 & & & \\
\hline & & 21 & & & & & 21 & 3 & 8 \\
\hline & & & & & & & 1 & & \\
\hline & & 12 & & & & & 8 & & 10 \\
\hline & & 3 & & & 1 & 2 & & 1 & \\
\hline & & 11 & & & & & 1 & & 4 \\
\hline & & 7 & & & & & 5 & & \\
\hline & & & & & & & 6 & 1 & 2 \\
\hline & & & 1 & & & 2 & & & \\
\hline & & & & & & 3 & 1 & & \\
\hline & & 3 & & 1 & & & 15 & & 1 \\
\hline 2 & & 1 & & & & 4 & & 1 & \\
\hline & & & & & 1 & & 1 & & 2 \\
\hline & & & & & & & & & \\
\hline & & 10 & 1 & & & 3 & 3 & 2 & 3 \\
\hline & & 1 & & & & & 3 & & \\
\hline & & 2 & & & & & & 3 & \\
\hline & & & & & & & 1 & & 1 \\
\hline 3 & & 9 & & & & 1 & 2 & 2 & 5 \\
\hline 6 & & 82 & 2 & 2 & 3 & 17 & 74 & 12 & 39 \\
\hline 349 & 336 & 244 & 344 & 346 \\
\hline
\end{tabular}

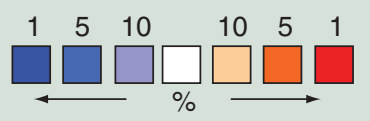

Figure 1. The mRNA levels of MFAPs in different cancer types. The data were collected from Oncomine cancer profiling database (www.oncomine.org/) in cancer tissues compared with normal tissues. The gene rank was analyzed by percentile of target gene in the top of all genes measured in each research. Red and blue color scales determined by the best gene rank percentile indicate gene expression up-regulation and down-regulation, respectively. The cut-off of $p$-value, fold change and gene ranking were defined as $0.01,2$ and $10 \%$ respectively.

\section{Results}

\section{MFAPs-mRNA expression in breast cancer}

To investigate the roles of MFAPs in human carcinogenesis, we analyzed the mRNA expression of MFAPs in a wide variety of cancer types represented in the Oncomine databases. The mRNA expression levels of MFAP2, MFAP4 and MFAP5 were abnormally expressed in most human cancers, among which breast cancer has the most data available (Figure 1). Compared with normal tissues, MFAP2 were significantly upregulated in breast cancer (21 datasets). Meanwhile, MFAP4 and MFAP5 were obviously downregulated in breast cancer tissues compared with those in normal tissues. However, in Finak's datasets, MFAP5 was significantly increased in invasive breast carcinoma with a 3.746-fold change [19]. Oncomine data also revealed an increase of MFAP1 and MFAP3-mRNA in breast cancer compared with those in normal tissues $(\mathrm{p}<0.01)$ (Table 1$)$.

Next, we confirmed the expression of MFAPs using UALCAN. We found that MFAP1, MFAP2, MFAP4 and MFAP5 were significantly dysregulated in breast cancer. Among them, MFAP2 was upregulated 4.80-fold ( $\mathrm{p}=$ 1.62e-12) (Figure 2B), while MFAP4 and MFAP5 were greatly downregulated 8.59-fold and 3.18-fold, respectively $(\mathrm{p}<1.00 \mathrm{e}-12$ and $\mathrm{p}=3.46 \mathrm{e}-10)$ (Figure $2 \mathrm{D} \& 2 \mathrm{E})$. The mRNA expression of MFAP1 was just slightly increased 1.04-fold in breast cancer ( $\mathrm{p}=0.0024$ ) (Figure 2A). MFAP3 was upregulated 1.14-fold and there was no significant difference of MFAP3 between breast cancer tissues and normal tissues ( $\mathrm{p}=0.1532$ ) (Figure 2C). 

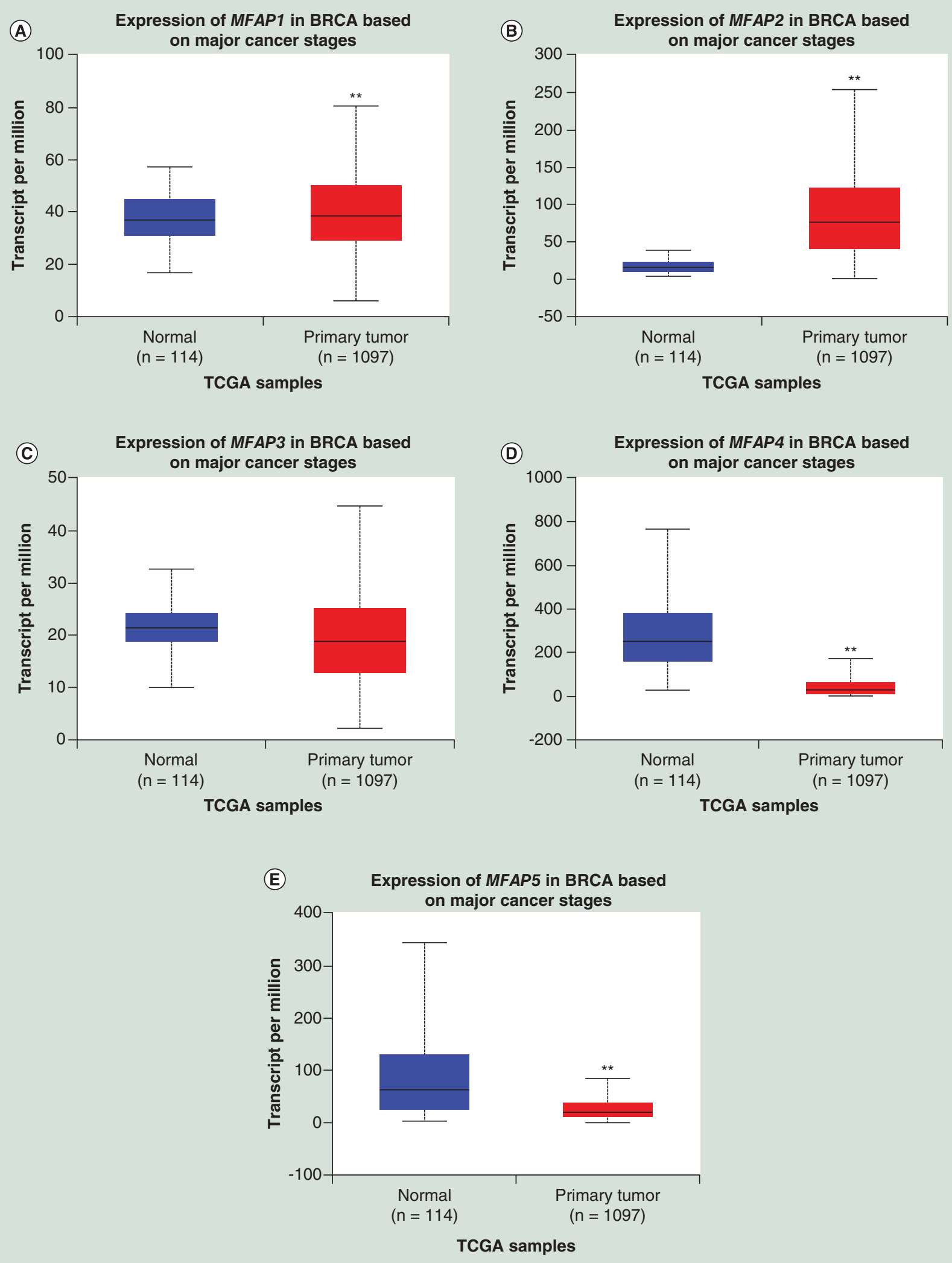

Figure 2. Box-whisker plots showing the mRNA levels of MFAPs in breast invasive carcinoma samples based on major cancer stages from UALCAN database. (A) MFAP1; (B) MFAP2; (C) MFAP3; (D) MFAP4; (E) MFAP5.

$* * p<0.01$. 
Table 1. The significant changes of microfibrillar-associated proteins-mRNA expression between different types of breast cancer and breast tissues in Oncomine database.

\begin{tabular}{|c|c|c|c|c|c|}
\hline Gene name & Types of breast cancer vs normal breast tissues & Fold change & p-value & t-test & Ref. \\
\hline \multirow[t]{2}{*}{ MFAP1 } & Tubular breast carcinoma & 1.527 & 7.63E-31 & 15.791 & [20] \\
\hline & Mucinous breast carcinoma & 1.557 & 4.76E-18 & 12.072 & \\
\hline MFAP2 & Invasive ductal breast carcinoma & 4.265 & $6.80 \mathrm{E}-05$ & 4.571 & [21] \\
\hline \multirow[t]{4}{*}{ MFAP3 } & Ductal breast carcinoma in situ & 1.252 & 4.10E-05 & 5.754 & [20] \\
\hline & Benign breast neoplasm & 1.290 & 0.007 & 5.883 & \\
\hline & Invasive ductal and invasive lobular breast carcinoma & 1.311 & $3.51 \mathrm{E}-19$ & 10.061 & \\
\hline & Breast carcinoma & 1.208 & $3.90 \mathrm{E}-4$ & 4.120 & \\
\hline \multirow[t]{5}{*}{ MFAP4 } & Mucinous breast carcinoma & -16.406 & $1.07 \mathrm{E}-14$ & -23.455 & TCGA \\
\hline & Invasive ductal breast carcinoma & -8.969 & $8.59 \mathrm{E}-36$ & -20.036 & \\
\hline & Invasive breast carcinoma & -3.752 & 4.11E-18 & -9.952 & \\
\hline & Invasive lobular breast carcinoma & -2.971 & $3.21 \mathrm{E}-9$ & -6.649 & \\
\hline & Intraductal cribriform breast adenocarcinoma & -6.842 & 0.008 & -6.184 & \\
\hline \multirow[t]{2}{*}{ MFAP5 } & Invasive breast carcinoma & 3.746 & $9.66 \mathrm{E}-23$ & 17.24 & [19] \\
\hline & Invasive breast carcinoma & -1.718 & $4.30 \mathrm{E}-5$ & -6.146 & [22] \\
\hline
\end{tabular}

Prognostic values of MFAPs in all breast cancer patients

Analysis of 1402 breast cancer in KM plotter showed that high mRNA levels of MFAP3 and MFAP4 were significantly associated with better prognosis (R: 0.64 [0.49-0.83] [Figure 3C \& 3D]; $\mathrm{p}=0.00081$ and HR: 0.52 [0.41-0.67]; $\mathrm{p}=1.4 \mathrm{e}-07)$. In addition, high mRNA levels of MFAP1 and MFAP2 were modestly associated with increased OS in breast cancer (HR: 0.81 [0.64-1.03]; $\mathrm{p}=0.081$ and HR: 0.81 [0.65-1.01]; $\mathrm{p}=0.059$ ) (Figure $3 \mathrm{~A}$ \& 3B). However, MFAP5 (HR: 1.18 [0.93-1.49]; $\mathrm{p}=0.17$ ) (Figure 3E) was not correlated with OS despite its significantly decreased mRNA expression. Considering their mRNA expression and association with OS, MFAP4 might provide higher prognostic values in clinical practice for breast cancer patients compared with other MFAPs family members.

\section{Prognostic values of MFAP4 in breast cancer patients with different intrinsic subtypes \& cancer grades}

To further examine the prognostic values of MFAP4, we first checked mRNA expression of MFAP4 in different major subclasses of TCGA breast cancer samples using UALCAN database. As shown in Figure 4A, mRNA levels of MFAP4 were significantly reduced in luminal, HER2 positive and triple negative breast cancers compared with normal tissues $(\mathrm{p}=1.62 \mathrm{e}-12 ; \mathrm{p}=1.62 \mathrm{e}-07$ and $\mathrm{p}<1.00 \mathrm{e}-12$; respectively). Then we assessed prognostic values of MFAP4 in different intrinsic subtypes using KM plotter. The results showed high mRNA expression of MFAP4 in all four intrinsic subtypes significantly correlated with better prognosis (Basal; HR: 0.53 [0.32-0.90]; $\mathrm{p}=0.016$; Luminal A; HR: 0.42 [0.28-0.64] [Figure 4B-E]; $\mathrm{p}=$ 2.2e-05); Luminal B; HR: 0.66 (0.44-0.99); $\mathrm{p}=0.045$; HER2+, HR: $0.43(0.22-0.85) ; \mathrm{p}=0.012$, respectively.

We also investigated prognostic values of MFAP 4 in breast cancer patients with different cancer grades. UALCAN database analysis showed MFAP4 was frequently downregulated in all individual cancer stages (all $\mathrm{p}<0.01$ ) (Figure 4F). Further analysis revealed high mRNA expression of MFAP4 was correlated with better prognosis in grade II (HR: 0.45 [0.29-0.68]; $\mathrm{p}=0.00014$ ) (Figure 4H). Interestingly, high mRNA expression of MFAP4 was related to poor prognosis in grade III (HR: 1.6 [1.15-2.22]; $\mathrm{p}=0.0052$ ) (Figure 4I). However, there was no significant correlation of high mRNA expression of MFAP4 in grade I and OS (HR: 0.49 [0.2-1.19]; $\mathrm{p}=0.11$ ) (Figure $4 \mathrm{G}$ ). Taken together, these findings indicate a high prognostic value of MFAP4 for breast cancer patients.

\section{DNA hypermethylation in the promoter of MFAP4 negatively correlates with MFAP4-mRNA} expression in breast cancer

DNA methylation plays a critical role in the epigenetic regulation of chromatin structure and gene transcription and high levels of DNA methylation in promoter regions could directly lead to stable epigenetic repression of gene expression [23]. To assess whether abnormal expression of MFAP4 is associated with DNA methylation status, MEXPRESS analysis was used in this study. As shown in Figure 5A, the samples are ordered by their expression level. 

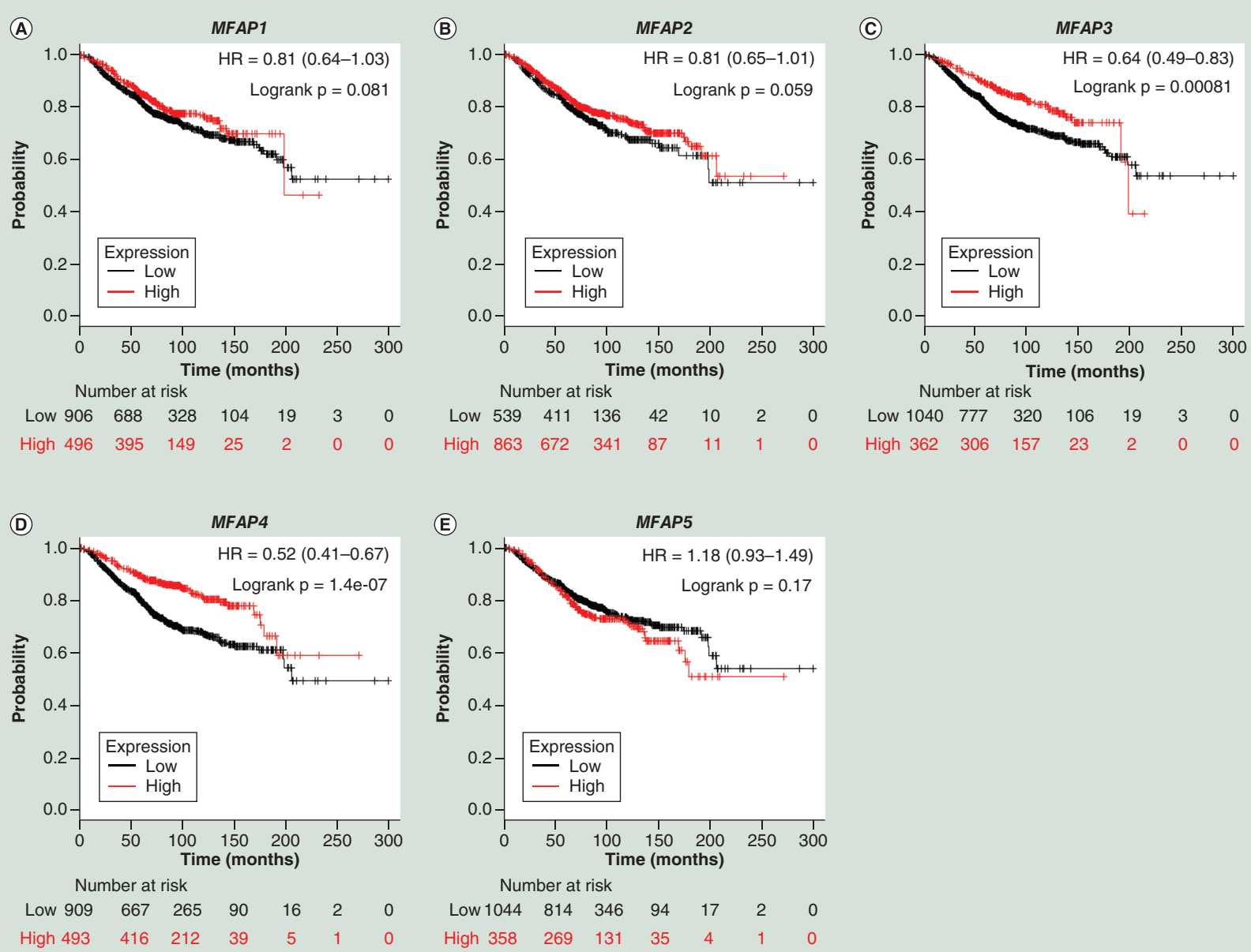

Figure 3. Prognostic values of MFAPs for OS in all breast cancer patients. The Kaplan-Meier plots were generated by analyzing 1105 breast invasive carcinoma patients (TCGA, Provisional, 1105 samples). p-values were calculated by log-rank test. HRs with $95 \%$ Cls are displayed. (A) MFAP1; (B) MFAP2; (C) MFAP3; (D) MFAP4; (E) MFAP5.

The expression of MFAP4 in breast cancer was negatively correlated with its DNA methylation in the promoter, which was confirmed by the Pearson correlation coefficients on the right (Figure 5A). MEXPRESS analysis also revealed that MFAP4-mRNA expression level was much lower in breast cancer samples than those in normal samples and correlated with $P R$ status $(\mathrm{p}=5.7 \mathrm{e}-07)$ and $E R$ status $(\mathrm{p}=5.62 \mathrm{e}-09)$. Data from the MethHC database confirmed DNA hypermethylation in the promoter of MFAP4 in breast cancer patients compared with normal samples $(\mathrm{p}<0.01)$ (Figure 5B). These results demonstrate that DNA hypermethylation in the promoter of MFAP4 may result in a downregulation of its mRNA expression.

We also determined whether decreased mRNA expression of MFAP4 was associated with genetic alterations, the cBioPortal was used to analyze mutations, copy-number alterations and mRNA expression changes of MFAP4. The alterations of MFAP4 were mutually exclusive, occurred in 57 (6\%) of 960 patients with breast invasive carcinoma, 17 samples of whom occurred DNA amplification, one sample was inframe mutated, eight samples were observed to have deep deletion and only 31 samples were mRNA upregulated (Figure 5C). Despite its DNA copy-number alteration, there was almost no elevation in the mRNA expression of MFAP4, but slightly decreased (Figure 5D).

\section{MFAP4-regulated biomolecular network}

To investigate how MFAP4 exerts its regulatory effect in the tumorogenesis and tumor progression, we searched its potential regulated genes using STRING. Functional interaction network analysis revealed that MFAP4 was closely associated with 35 genes, including FBLN1, FBLN5, EFEMP1, EFEMP2, MFAP2, MFAP5, PMP22 and COTL1 (Figure 6A). Then we performed gene ontology and pathway enrichment analysis for those genes using 


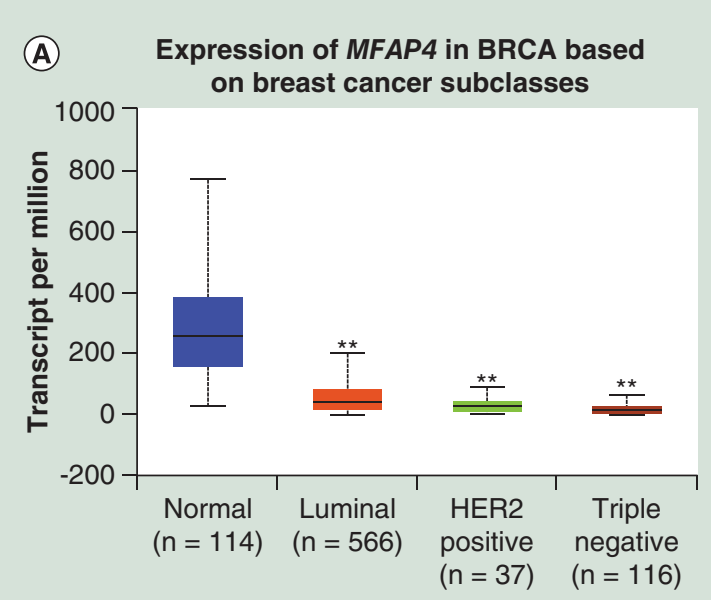

TCGA samples

(C)

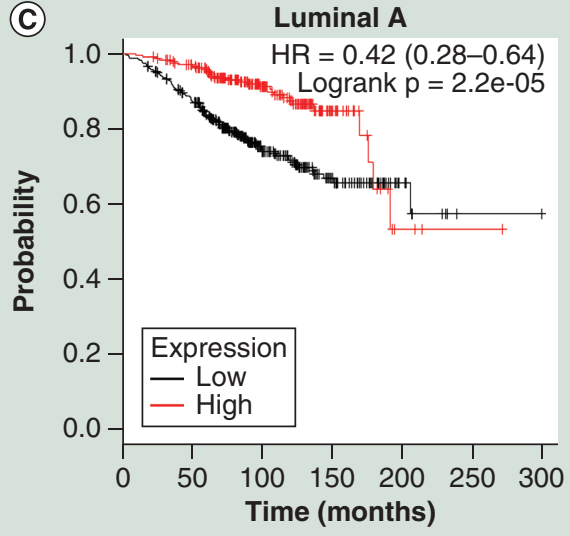

Number at risk

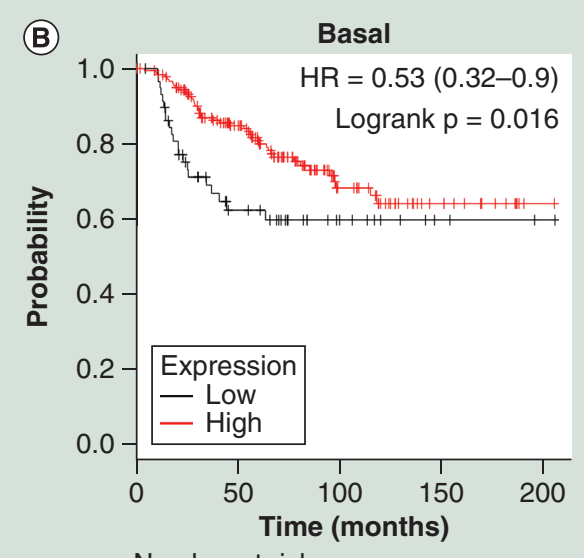

Number at risk

$\begin{array}{cccccc}\text { Low } & 59 & 26 & 10 & 3 & 1 \\ \text { High } & 182 & 114 & 40 & 14 & 1\end{array}$

(D)

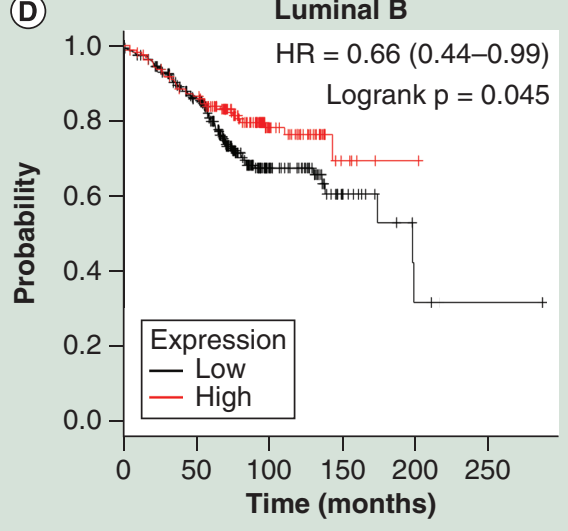

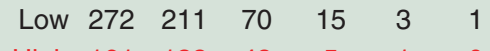

High $\begin{array}{lllllll}161 & 132 & 48 & 5 & 1 & 0\end{array}$

High $\begin{array}{lllllll}248 & 229 & 136 & 26 & 3 & 1 & 0\end{array}$

HER2+

(E)

$\mathrm{HR}=0.43(0.22-0.85)$

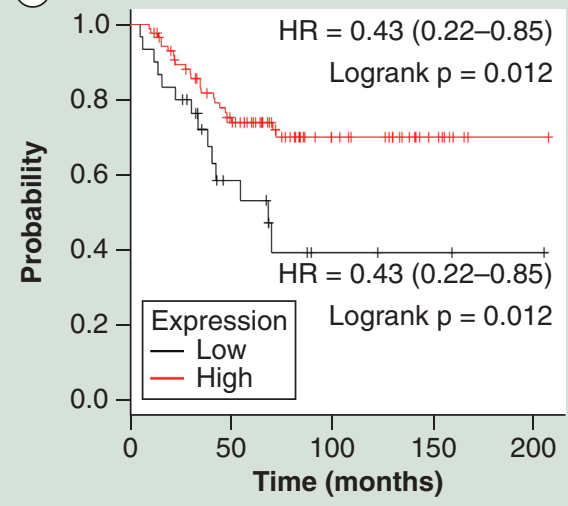

Number at risk

$\begin{array}{cccccc}\text { Low } & 30 & 11 & 3 & 2 & 1 \\ \text { High } & 87 & 56 & 23 & 8 & 1\end{array}$

Figure 4. Prognostic values of MFAP4 in breast cancer patientswith different intrinsic subtypes and cancer grades. (A) mRNA expression of MFAP4 in breast cancer patients with different intrinsic subtypes, including luminal, HER2 positive and triple negative breast cancer patients. Then MFAP4 was plotted for different intrinsic subtypes of breast cancer. (B) Basal breast cancer; (C) Luminal A breast cancer; (D) Luminal B breast cancer; (E) HER2+ breast cancer; (F) mRNA expression of MFAP4 in breast cancer patients with different cancer stages. Then prognostic values of MFAP4 were plotted for different cancer grades of breast cancer; (G) Stage 1; (H) Stage 2; (I) Stage 3. $* * p<0.01$. 

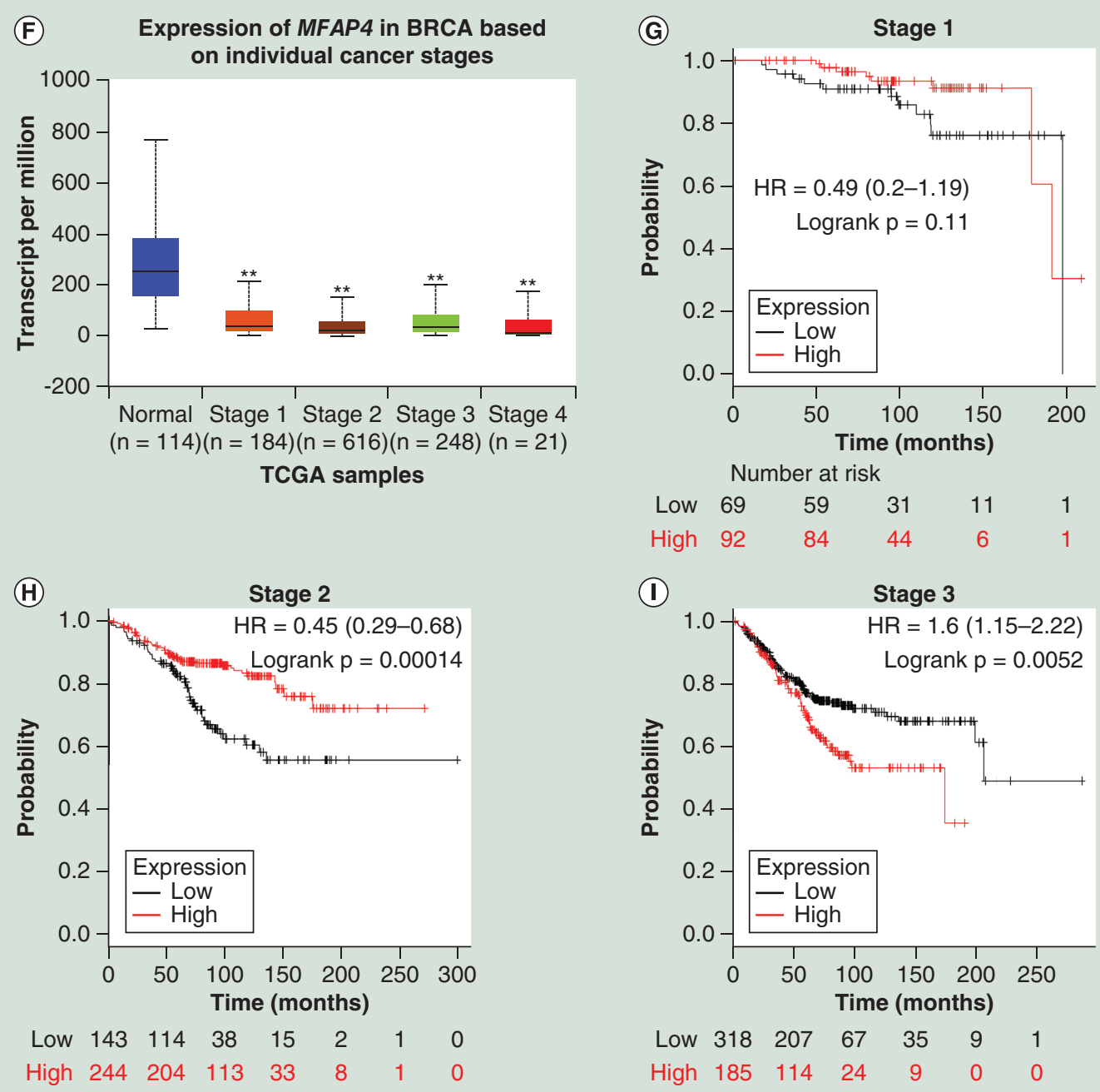

Figure 4. Prognostic values of MFAP4 in breast cancer patientswith different intrinsic subtypes and cancer grades (cont.). (A) mRNA expression of MFAP4 in breast cancer patients with different intrinsic subtypes, including luminal, HER2 positive and triple negative breast cancer patients. Then MFAP4 was plotted for different intrinsic subtypes of breast cancer. (B) Basal breast cancer; (C) Luminal A breast cancer; (D) Luminal B breast cancer; (E) HER2+ breast cancer; (F) mRNA expression of MFAP4 in breast cancer patients with different cancer stages. Then prognostic values of MFAP4 were plotted for different cancer grades of breast cancer; (G) Stage 1; (H) Stage 2; (I) Stage 3. $* * p<0.01$.

Metascape and the most significantly enriched gene sets were molecules associated with elastic fibres (FBLN1, FBLN2, FBN1, EFEMP1, MFAP2, MFAP5, FBLN5, EFEMP2, DCN) and nucleotide-excision repair (NER), DNA damage recognition (GPS1, UBB, COPS3, COPS2, COPS8, SHMT2, GJBG, FBN1, DCN and NEURL1) (Figure 6B \& Supplementary Table 2). In addition, signaling by NOTCH1 HD domain mutants in cancer (UBB, NEURL1, NEURL1B, EFEMP1, OCA2, SLC26A8 and DCN), PID Notch pathway (MFAP2, MFAP5, NEURL1) and negative regulation of cell proliferation (FBLN1, PMP22, SFTPD, NEURL1, GJBG, COPS8 and FBN1) were also significantly enriched, which have been reported as pivotal biological process in tumor progression. These results indicated that downregulation of MFAP4 in breast cancer may play an important role in cell proliferation and impairment and alteration of the elastic fibers structural appearance.

Prognostic values of MFAP4 in other human cancers

According to the latest cancer situation and trend from the Chinese National Cancer Center, lung cancer, liver cancer and stomach cancer are top three cancers with the highest morbidity and mortality in China. We decided to test 


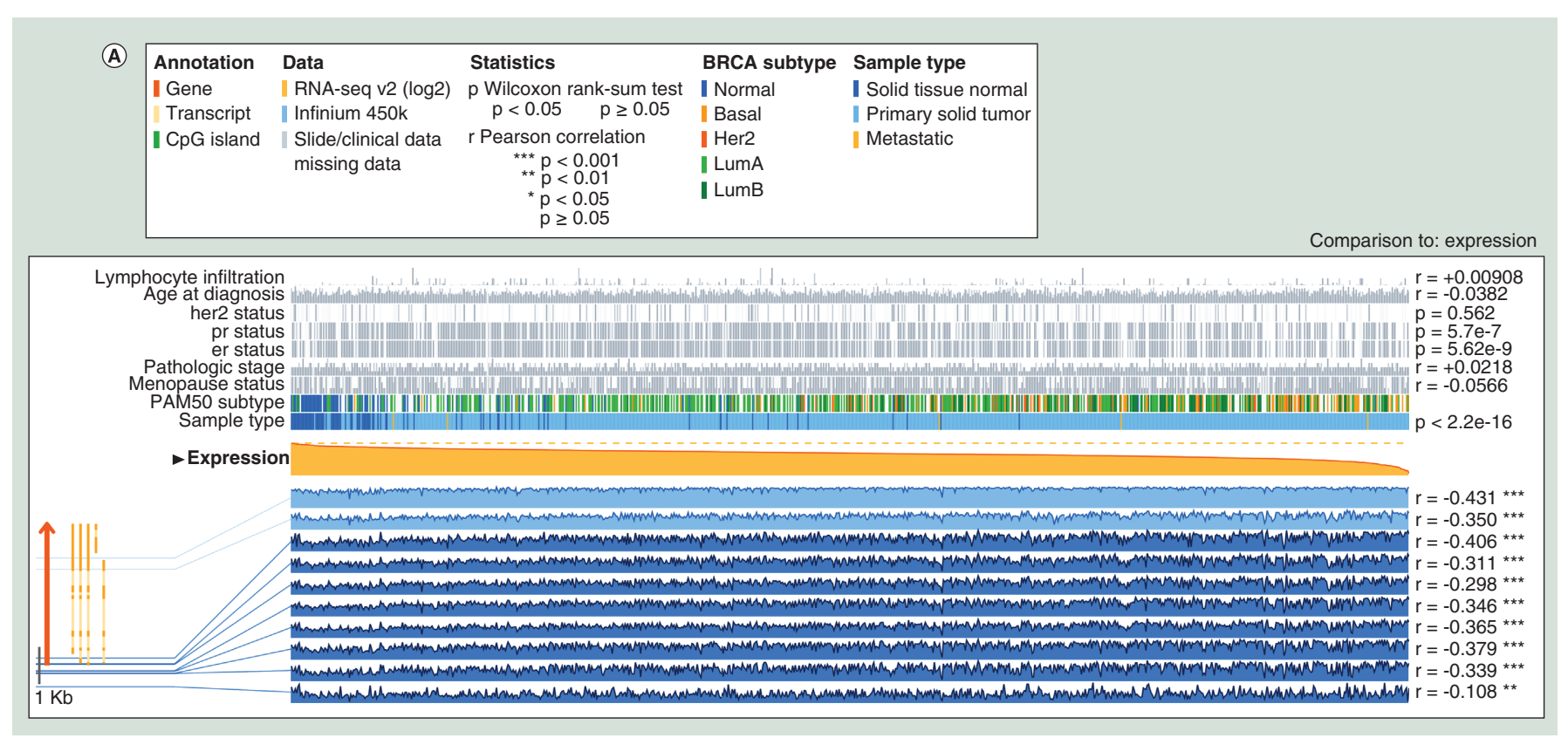

Figure 5. Impact of epigenetic and genetic alterations on MFAP4 mRNA expression in breast cancer. (A) Visualization of the TCGA data for MFAP4 mRNA expression in breast cancer using MEXPRESS. 871 breast invasive carcinoma samples were included. The methylation data for each probe was determined using Infinium HumanMethylation 450 microarray. Gene expression data was derived from IlluminaHiSeq_RNASeqV2. The samples are ordered by their expression level. The significance of the relation (correlation coefficient or p-value) between each row of data (clinical, expression or methylation) was shown in the right side. (B) The promoter methylation levels of MFAP4 in 839 breast invasive carcinoma samples were analyzed by MethHC database. (C) OncoPrint of MFAP4 alterations in breast cancer. Tumor samples are shown in columns. Genomic alterations of MFAP4 are mutually exclusive. (D) Correlation plot for putative copy-number alterations and MFAP4 mRNA expression in breast cancer. Putative copy-number calls on 1080 breast cancer cases determined using GISTIC 2.0. Values: -2 = homozygous deletion; $-1=$ hemizygous deletion; $0=$ neutral $/$ no change; $1=$ gain; $2=$ high level amplification.

${ }^{*} \mathrm{p}<0.05 ; * * \mathrm{p}<0.01 ; * * * \mathrm{p}<0.001$.

the prognostic values of MFAP4 in these human cancers. UALCAN analysis showed a significantly downregulated mRNA level of MFAP4 in cancer tissues compared with normal tissues (Figure 7A-C). KM plotter database revealed high mRNA expression of MFAP4 were significantly correlated with better outcome in all lung cancer patients (HR: 0.58 [0.51-0.67]; $\mathrm{p}=5.4 \mathrm{e}-15$ ) (Figure 7D), liver cancer patients (HR: 0.63 [0.42-0.93]; $\mathrm{p}=0.02$ ) (Figure 7E) and stomach cancer patients (HR: 0.72 [0.59-0.88]; $\mathrm{p}=0.0013$ ) (Figure 7F). We also investigated its prognostic values in lung, liver and stomach cancer patients with different stages. As shown in Table 2 and Supplementary Figure 1, MFAP4 presented a good prognostic values for these three cancer patients in most cases.

\section{Discussion}

Seeking for useful biomarkers and novel therapeutic targets represent an important milestone toward precision oncology for diagnosis and treatment of human cancers. The present study systematically profiled the global landscape of mRNA expression, prognostic values, epigenetic and genetic alterations and regulatory network for MFAPs in human cancers. Using breast cancer as a study model, we identified a frequent downregulation of MFAP4 in most human cancers and its high mRNA level predicts a better prognosis for breast cancer patients with different intrinsic subtypes and cancer grades, except for stage III. Consistent with that of breast cancer, high mRNA levels of MFAP4 in lung, liver and stomach cancers are also significantly associated with better outcome in early stages. Compared with established prognostic biomarker for breast cancer, such as cancer antigen 15-3 (CA 15-3), CA 125, carcinoembryonic antigen, ER, PR and HER2, MFAP 4 seems to be a common prognostic biomarker in most types of human cancers. These findings may contribute to the development of precise diagnosis and prognosis based on MFAP4 in human cancers. Increased DNA copy number always means an increase of gene mRNA expression level. But several biological factors like mutation, DNA methylation, histone modifications and microRNAs could significantly affect gene mRNA expression beyond copy number itself [24]. In this study, hypermethylation in 


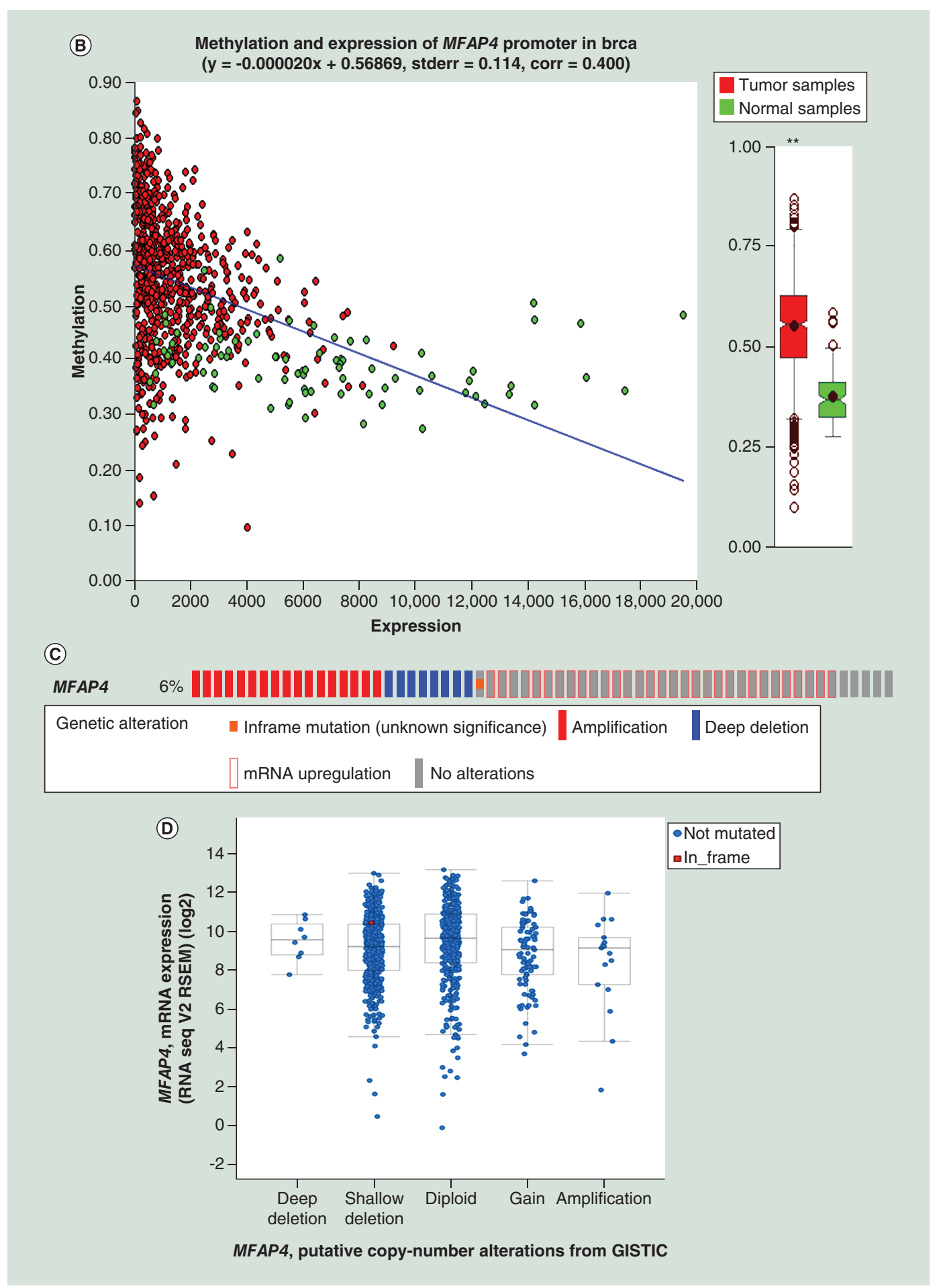

Figure 5. Impact of epigenetic and genetic alterations on MFAP4 mRNA expression in breast cancer (cont.). (A) Visualization of the TCGA data for MFAP4 mRNA expression in breast cancer using MEXPRESS. 871 breast invasive carcinoma samples were included. The methylation data for each probe was determined using Infinium HumanMethylation 450 microarray. Gene expression data was derived from IlluminaHiSeq_RNASeqV2. The samples are ordered by their expression level. The significance of the relation (correlation coefficient or p-value) between each row of data (clinical, expression or methylation) was shown in the right side. (B) The promoter methylation levels of MFAP4 in 839 breast invasive carcinoma samples were analyzed by MethHC database. (C) OncoPrint of MFAP4 alterations in breast cancer. Tumor samples are shown in columns. Genomic alterations of MFAP4 are mutually exclusive. (D) Correlation plot for putative copy-number alterations and MFAP4 mRNA expression in breast cancer. Putative copy-number calls on 1080 breast cancer cases determined using GISTIC 2.0. Values: $-2=$ homozygous deletion; -1 = hemizygous deletion; 0 = neutral/no change; 1 = gain; 2 = high level amplification. ${ }^{*} \mathrm{p}<0.05 ;{ }^{*} \mathrm{p}<0.01 ;{ }^{* *} \mathrm{p}<0.001$. 
(A)

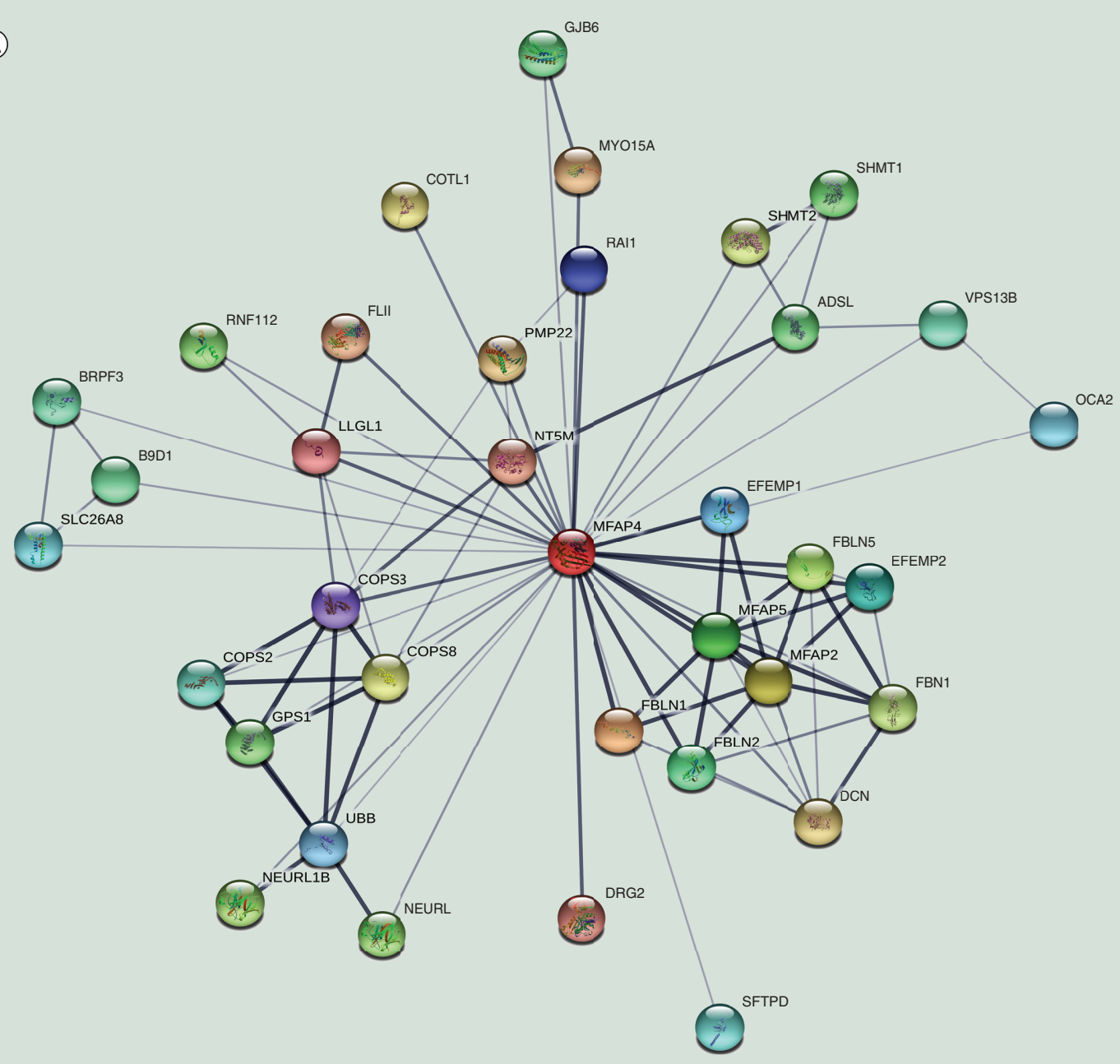

(B)

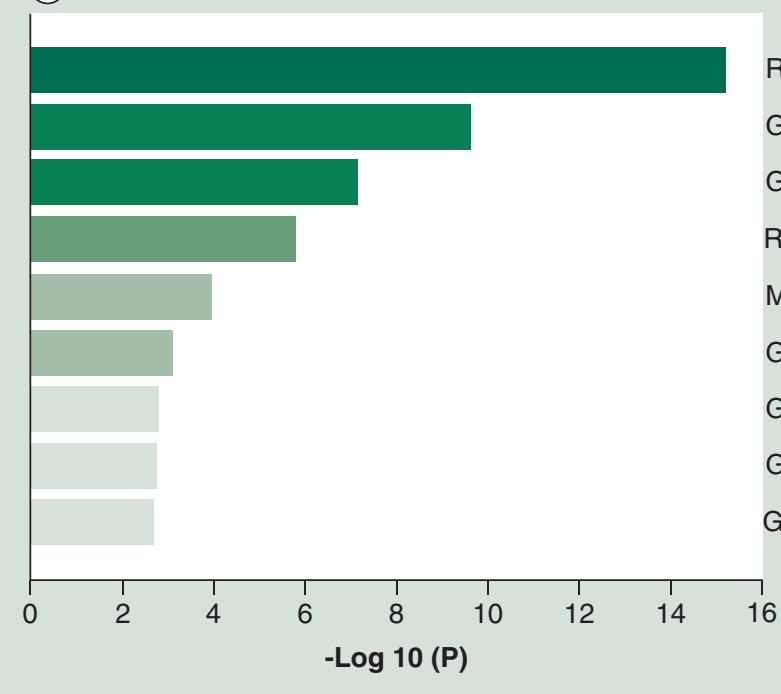

Figure 6. MFAP4-regulated biomolecular network. (A) Protein-protein interaction networks were analyzedby STRING database with a confidence score of 0.4 and 35 genes interacted with MFAP4 were identified. Line thickness indicates the strength of data

support. (B) Top 9 clusters from Metascape pathway enrichment analysis of 35 MFAP4-associated genes carried out with the following ontology sources: KEGG Pathway, GO Biological Processes, Reactome Gene Sets, Canonical Pathways and CORUM. Length of bars represent $\log 10$ ( $p$-value) based on the best-scoring term within each cluster. 

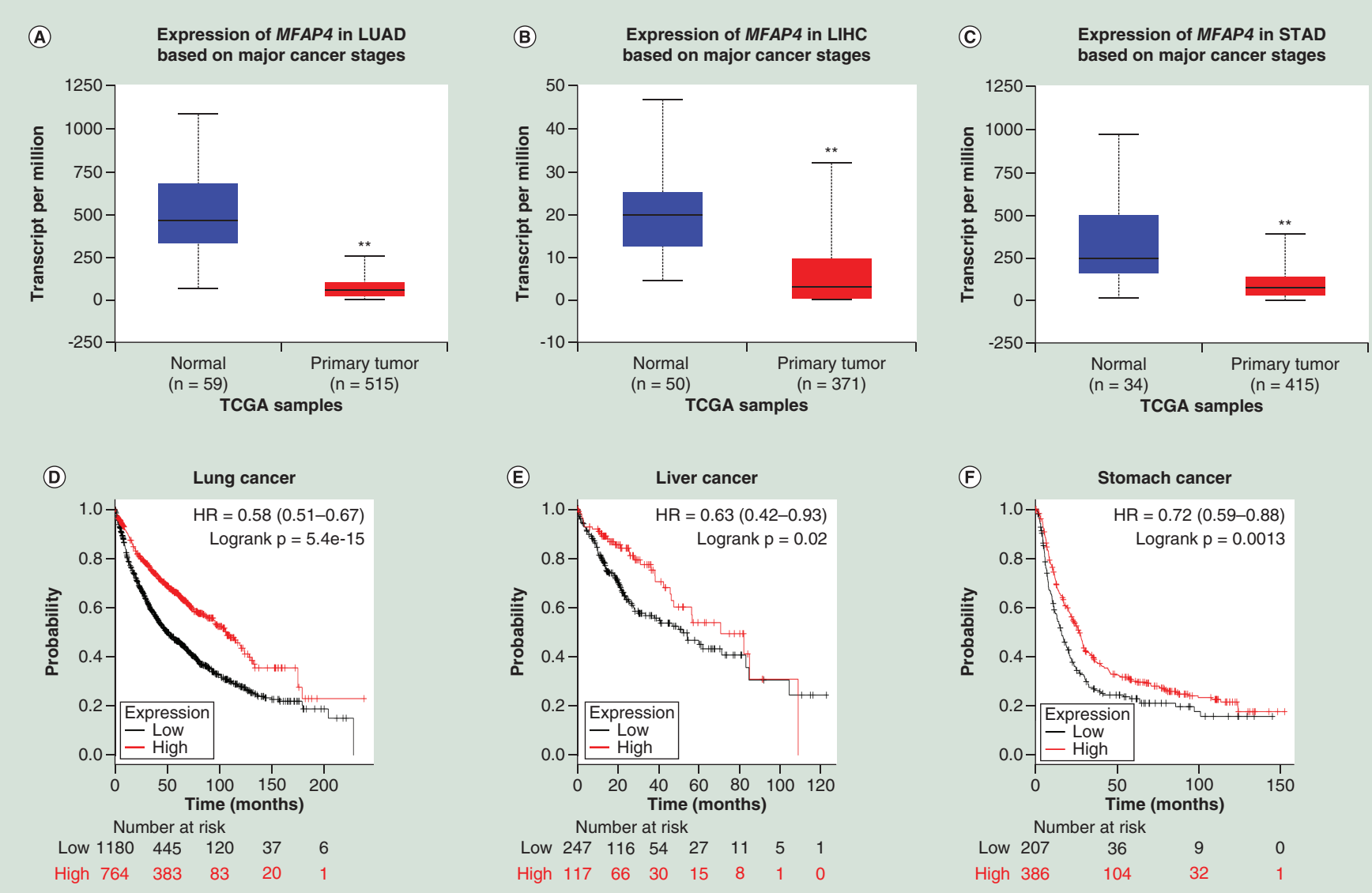

Figure 7. Prognostic values of MFAP4 in other human cancers. MFAP4 mRNA expression was analyzed in 574 lung cancer cases, 421 liver cancer cases and 449 stomach cancer cases from UALCAN database. (A) Expression of MFAP4 in lung cancer. (B) Expression of MFAP4 in liver cancer. (C) Expression of MFAP4 in stomach cancer. Then we analyzed prognostic values of MFAP4 in these cancers using KM plotter. (D) MFAP4 in all lung cancer patients. (E) MFAP4 in all liver cancer patients. (F) MFAP4 in all stomach cancer patients. p-values were calculated by log-rank test. HRs with $95 \% \mathrm{Cls}$ are displayed.

$* * p<0.01$.

the promoter may be a key drive for repression of MFAP4 transcription and mRNA expression. Meanwhile, the mutations and copy-number alterations have little impact on gene transcription for MFAP4.

MFAP4, a human homolog to MAGP-36, is expressed in both elastic and nonelastic tissues [25-27]. MFAP4 was reported to be involved in immune response, skin disease, lung function and liver fibrosis [28-31]. Several studies have also indicated that MFAP4 may play potential roles in human cancers. For example, MFAP4 was downregulated and may function as tumor suppressor in prostate cancer and urinary bladder cancer [32,33]. Consistent with our results, Muraoka et al. found that MFAP4 protein level was higher in low-risk breast cancer patients than those of high-risk patients, but the function of MFAP4 in breast cancer was not clarified [34]. In neuroblastoma, however, Zhao et al. reported that miR-449a inhibited cell proliferation and induced cell-cycle arrest by targeting MFAP4, PKP4 and TSEN15 and high level of MFAP4 was correlated with poor survival of neuroblastoma patients [35]. Similar to neuroblastoma, here we also observed that high level of MFAP4 predicted a poor prognosis for patients with advanced stages of lung and stomach cancer as well as breast cancer. This apparent shift of MFAP4 in prognostic ability probably results from a dual role of MFAP4. In early stage cancer, MFAP4 facilitates inflammatory cell recruitment and assists immunological cancer surveillance to restrain cancer-cell survival [28]. In late stage cancer, alteration of tumor microenvironment results in decreased immune function of lymphocytes and MFAP4 predominantly promotes cancer cell proliferation and migration and therefore, accelerates tumor exacerbation [36]. As the physiological and pathophysiological roles of MFAP4 are still largely unknown, further 


\begin{tabular}{|c|c|c|c|c|c|c|c|}
\hline Cancer type & $\begin{array}{l}\text { Patients number } \\
\text { at risk }\end{array}$ & $\begin{array}{l}\text { MFAP4 expression } \\
\text { level }\end{array}$ & Stage & HR & $95 \% \mathrm{Cl}$ & $\begin{array}{l}\text { Prognostic } \\
\text { outcome }\end{array}$ & p-value \\
\hline \multirow[t]{8}{*}{ Lung cancer } & 363 & Low & 1 & 0.28 & $0.19-0.4$ & Good & $4.4 \mathrm{e}-13$ \\
\hline & 214 & High & & & & & \\
\hline & 144 & Low & 2 & 0.65 & $0.44-0.95$ & Good & 0.026 \\
\hline & 100 & High & & & & & \\
\hline & 4 & - & 4 & - & - & - & - \\
\hline & 42 & Low & 1 & 0.45 & $0.23-0.89$ & Good & 0.019 \\
\hline & 128 & High & & & & & \\
\hline & 27 & Low & 2 & 0.53 & $0.24-1.16$ & Good & 0.11 \\
\hline \multirow{3}{*}{ Liver cancer } & 16 & Low & 1 & 0.52 & $0.17-1.6$ & Good & 0.25 \\
\hline & 23 & High & & & & & \\
\hline & 11 & Low & 2 & 2.72 & $0.64-11.66$ & Worse & 0.16 \\
\hline \multirow[t]{5}{*}{ Stomach cancer } & 38 & High & & & & & \\
\hline & 62 & Low & 3 & 1.39 & $0.96-2.03$ & Worse & 0.082 \\
\hline & 155 & High & & & & & \\
\hline & 26 & Low & 4 & 1.37 & $0.75-2.49$ & Worse & 0.3 \\
\hline & 48 & High & & & & & \\
\hline
\end{tabular}

studies are needed to determine the protein levels of MFAP4 in different breast cancer stages, which is important for us to explain the obvious distinction of MFAP4 in prognostic ability in late stage cancer.

By browsing networks for genes of interest, especially rare reported genes, we can predict its potential functions and identify a number of previously unappreciated biological processes and pathways associated with human diseases. Functional enrichment analysis of 35 MFAP4-related genes resulted in the identification of remarkable pathways which may play key roles in cancer initiation and progression. As expected, genes that associate with elastic fibres were significantly enriched. More importantly, newly identified MFAP4-related pathways, NER and negative regulation of cell proliferation will encourage future investigations which may contribute to the discovery of novel mechanisms governing carcinogenesis and tumor progression.

The accumulated DNA damage is an important driving force for cancer initiation. Endogenously metabolic products (such as reactive oxygen species) and environmental chemicals (tobacco) and radiation (ultraviolet) can generate single nucleotide mutations and DNA strand breaks [37-39]. These damages may activate oncogenes or inactivate tumor suppressor genes and cause abnormal cell growth, invasion and metastasis. NER is the most versatile system to eliminate diverse DNA lesions [40]. A significant correlation has been reported between polymorphisms in NER genes and a group of cancers such as breast, ovarian, lung and non-small-cell lung cancers [41-44]. Based on protein-protein interaction network in STRING database, MFAP4 might participate in NER process by direct or indirect interaction with NER proteins through predicted proteins such as SHMT2, COPS3 and COPS8, most of which have not yet been studied. In fact, these genes play important roles in diverse biological processes in human cancers. Kim et al. reported that SHMT2 desuccinylation at lysine 280 by SIRT5 increased enzymatic activity and promoted tumor cell growth [45]. COPS3 have been shown frequently upregulated and promoted cell proliferation, metastasis and apoptosis in clear cell renal cell carcinoma, osteogenic sarcoma and lung cancer [46-48].

Another identified important pathway related to MFAP4 was negative regulation of cell proliferation. FBLN1 belongs to extracellular matrix protein and is epigenetically downregulated in bladder cancer. Overexpression of FBLN1 could significantly suppress tumor growth and induce tumor cell apoptosis [49]. PMP22 was frequently amplified and upregulated in high-grade osteosarcoma and overexpression of PMP22 significantly promoted the proliferation, invasion, migration of osteosarcoma cells [50,51]. In this study, Notch signaling pathway was also 
enriched which is critical for cell proliferation, differentiation, development and homeostasis [52]. Dysregulation of the Notch signaling pathway has been implicated in a variety of hematologic and solid malignancies [53]. The study identified three genes (MFAP2, MFAP5 and NEURL1) associated with this pathway. MFAP2 was reported a crucial role in ECM function, cell adhesion, hemostasis, thrombosis and metabolic stress [54-56]. MFAP5 is a protein that can be secreted by cancer cells and promotes angiogenesis in cholangiocarcinoma and ovarian cancer [57,58]. MFAP2 and MFAP5 were also reported to possess the ability to interact with the EGF-like repeats of Notchs and subsequently activate Notch signaling pathway [59]. NEURL1 can function as an E3 ubiquitin ligase and decrease Notch ligand, Jagged 1 and the target genes, HES1 and HEY1 in medulloblastoma [60,61]. These results indicated a potential role of MFAP4 in the development and progression of human cancers.

One limitation of this study is that all our analysis was based on mRNA level from publicly available datasets and further investigations should be performed to validate the consistence with protein level by Western blot and immunohistochemistry staining. Although our work emphasizes the prognostic value of MFAP4 in human cancers and predicts several pathways involved in its function, experimental data are warranted to clarify the specific roles and underlying mechanisms through which MFAP4 influences cancer phenotypes such as cell growth, metastasis and apoptosis. Besides MFAP4 is an oligomeric glycoprotein containing several disulphide bridges [62]. That kind of structure is usually not found within cells, only in the extracellular milieu. It is of great interest to find how MFAP4 directly or indirectly participates in intracellular excision repair and DNS damage recognition.

\title{
Conclusion
}

In summary, our work for the first time systematically investigated the potential function and molecular mechanism of MFAPs in human cancers based on TCGA data. This integrated study revealed a profound impact of genetic and epigenetic alterations on gene expression in cancers. Our findings suggested MFAP4 may function as a candidate tumor suppressor gene and its potential roles in carcinogenesis is worthy of further study. Furthermore, MFAP4 could be used as a promising prognostic biomarker and potential novel therapeutic target against human cancers.

\section{Summary points}

- We investigated the expression profiles of MFAPs in different cancer types using Oncomine and UALCAN databases and found MFAP4 was frequently down-regulated.

- We found that high mRNA expression of MFAP4 was identified to significantly correlate with better overall survival in breast cancer.

- Epigenetic and genetic analysis revealed DNA hypermethylation in the promoter of MFAP4 decreased its mRNA expression in breast cancer.

- We demonstrated that MFAP4 strongly associated with pathways in impairment and alteration of the elastic fibers, nucleotide-excision repair and negative regulation of cell proliferation.

- MFAP4 was also confirmed high prognostic values in lung, liver and stomach cancers.

\author{
Supplementary data \\ To view the supplementary data that accompany this paper please visit the journal website at: \\ https://www.futuremedicine.com/doi/suppl/10.2217/epi-2018-0080
}

Author's contributions

Y Li and X Hao conceived and designed the study. J Yang, H Song, L Chen and Y Zhang obtained data. J Yang, H Song and K Cao performed the data analysis. J Yang and $\mathrm{H}$ Song drafted the manuscript. All authors reviewed the manuscript before submission. All the authors approved the final version of the manuscript.

Financial \& competing interests disclosure

This work was supported by grants from the National Natural Science Foundation of China (grant no. 81700169), the Natural Science Foundation of Guizhou Province (grant no. 201842920480710623), the 'Light of the West' Talent Cultivation Program of the Chinese Academy of Sciences (grant no. 201684) and the Found for Guizhou Fourth Batch of '100 Talented Leaders'. The authors have no other relevant affiliations or financial involvement with any organization or entity with a financial interest in or financial conflict with the subject matter or materials discussed in the manuscript apart from those disclosed.

No writing assistance was utilized in the production of this manuscript. 


\section{Open access}

This work is licensed under the Attribution-NonCommercial-NoDerivatives 4.0 Unported License. To view a copy of this license, visit http://creativecommons.org/licenses/by-nc-nd/4.0/

\section{References}

1. Halper J, Kjaer M. Basic components of connective tissues and extracellular matrix: elastin, fibrillin, fibulins, fibrinogen, fibronectin, laminin, tenascins and thrombospondins. Adv. Exp. Med. Biol. 802(11), 31-47 (2014).

2. Muiznieks LD, Weiss AS, Keeley FW. Structural disorder and dynamics of elastin. Biochem. Cell Biol. 88(2), 239-250 (2010).

3. Baldwin AK, Simpson A, Steer R et al. Elastic fibres in health and disease. Expert Rev. Mol. Med. 15(19), e8 (2013).

4. Mecham RP, Gibson MA. The microfibril-associated glycoproteins (MAGPs) and the microfibrillar niche. Matrix Biol. 47, 13-33 (2015).

5. Lausen M, Lynch N, Schlosser A et al. Microfibril-associated protein 4 is present in lung washings and binds to the collagen region of lung surfactant protein D. J. Biol. Chem. 274(45), 32234-32240 (1999).

6. Andersen DS, Tapon N. Drosophila MFAP1 is required for pre-mRNA processing and G2/M progression. J. Biol. Chem. 283(45), 31256-31267 (2008).

7. Ulrich AK, Wahl MC. Human MFAP1 is a cryptic ortholog of the Saccharomyces cerevisiae Spp381 splicing factor. BMC Evol. Biol. 17(1), 91 (2017).

8. Rode S, Ohm H, Zipfel J et al. The spliceosome-associated protein Mfap1 binds to VCP in Drosophila. PLoS ONE 12(8), e0183733 (2017).

9. Lou X, Kang B, Zhang J et al. MFAP3L activation promotes colorectal cancer cell invasion and metastasis. Biochim. Biophys. Acta 1842(9), 1423-1432 (2014).

10. Rhodes DR, Yu J, Shanker K et al. ONCOMINE: a cancer microarray database and integrated data-mining platform. Neoplasia 6(1), $1-6(2004)$.

11. Chandrashekar DS, Bashel B, Balasubramanya SAH et al. UALCAN: a portal for facilitating tumor subgroup gene expression and survival analyses. Neoplasia 19(8), 649-658 (2017).

12. Lánczky A, Nagy Á, Bottai G et al. miRpower: a web-tool to validate survival-associated miRNAs utilizing expression data from 2178 breast cancer patients. Breast Cancer Res. Treat. 160(3), 439-446 (2016).

13. Cerami E, Gao J, Dogrusoz U et al. The cBio cancer genomics portal: an open platform for exploring multidimensional cancer genomics data. Cancer Discov. 2(5), 401-404 (2012).

14. Mermel CH, Schumacher SE, Hill B et al. GISTIC2.0 facilitates sensitive and confident localization of the targets of focal somatic copy-number alteration in human cancers. Genome Biol. 12(4), R41 (2011).

15. Koch A, De Meyer T, Jeschke J et al. MEXPRESS: visualizing expression, DNA methylation and clinical TCGA data. BMC Genomics 16, 636 (2015).

16. Huang WY, Hsu SD, Huang HY et al. MethHC: a database of DNA methylation and gene expression in human cancer. Nucleic Acids Res. 43(Database issue), D856-D861 (2015).

17. Szklarczyk D, Franceschini A, Wyder S et al. STRING v10: protein-protein interaction networks, integrated over the tree of life. Nucleic Acids Res. 43(Database issue), D447-D452 (2015).

18. Tripathi S, Pohl MO, Zhou Y et al. Meta- and orthogonal integration of Influenza 'OMICs' data defines a role for UBR4 in virus budding. Cell Host Microbe 18(6), 723-735 (2015).

19. Finak G, Bertos N, Pepin F et al. Stromal gene expression predicts clinical outcome in breast cancer. Nat. Med. 14(5), 518-527 (2008).

20. Curtis C, Shah SP, Chin SF et al. The genomic and transcriptomic architecture of 2000 breast tumors reveals novel subgroups. Nature 486(7403), 346-352 (2012).

21. Turashvili G, Bouchal J, Baumforth K et al. Novel markers for differentiation of lobular and ductal invasive breast carcinomas by laser microdissection and microarray analysis. BMC Cancer 7, 55 (2007).

22. Glück S, Ross JS, Royce M et al. TP53 genomics predict higher clinical and pathologic tumor response in operable early stage breast cancer treated with docetaxel-capecitabine \pm trastuzumab. Breast Cancer Res. Treat. 132(3), 781-791 (2012).

23. Smith ZD, Meissner A. DNA methylation: roles in mammalian development. Nat. Rev. Genet. 14(3), 204-220 (2013).

24. Sharma S, Kelly TK, Jones PA. Epigenetics in cancer. Carcinogenesis 31(1), 27-36 (2010).

25. Kobayashi R, Mizutani A, Hidaka H. Isolation and characterization of a 36-kDa microfibril-associated glycoprotein by the newly synthesized isoquinolinesulfonamide affinity chromatography. Biochem. Biophys. Res. Commun. 198(3), 1262-1266 (1994).

26. Brandsma CA, van den Berge M, Postma DS et al. A large lung gene expression study identifying fibulin-5 as a novel player in tissue repair in COPD. Thorax 70(1), 21-32 (2015). 
27. Toyoshima T, Ishida T, Nishi $\mathrm{N}$ et al. Differential gene expression of 36-kDa microfibril-associated glycoprotein (MAGP-36/MFAP4) in rat organs. Cell Tissue Res. 332(2), 271-278 (2008).

28. Niu D, Peatman E, Liu H et al. Microfibrillar-associated protein 4 (MFAP4) genes in catfish play a novel role in innate immune responses. Dev. Comp. Immunol. 35(5), 568-579 (2011).

29. Kasamatsu S, Hachiya A, Fujimura $T$ et al. Essential role of microfibrillar-associated protein 4 in human cutaneous homeostasis and in its photoprotection. Sci. Rep. 1, 164 (2011).

30. Holm AT, Wulf-Johansson H, Hvidsten $S$ et al. Characterization of spontaneous air space enlargement in mice lacking microfibrillar-associated protein 4. Am. J. Physiol. Lung Cell Mol. Physiol. 308(11), L1114-L1124 (2015).

31. Sækmose SG, Mössner B, Christensen PB et al. Microfibrillar-associated protein 4: a potential biomarker for screening for liver fibrosis in a mixed patient cohort. PLoS ONE 10(10), e0140418 (2015).

32. Davalieva K, Kostovska IM1, Kiprijanovska $S$ et al. Proteomics analysis of malignant and benign prostate tissue by 2D DIGE/MS reveals new insights into proteins involved in prostate cancer. Prostate 75(14), 1586-1600 (2015).

33. Zaravinos A, Lambrou GI, Boulalas I et al. Identification of common differentially expressed genes in urinary bladder cancer. PLoS ONE 6(4), e18135 (2011).

34. Muraoka S, Kume H, Watanabe S et al. Strategy for SRM-based verification of biomarker candidates discovered by iTRAQ method in limited breast cancer tissue samples. J. Proteome Res. 11(8), 4201-4210 (2012).

35. Zhao Z, Ma X, Sung D et al. microRNA-449a functions as a tumor suppressor in neuroblastoma through inducing cell differentiation and cell cycle arrest. RNA Biol. 12(5), 538-554 (2015).

36. Schlosser A, Pilecki B, Hemstra LE et al. MFAP4 promotes vascular smooth muscle migration, proliferation and accelerates neointima formation. Arterioscler. Thromb. Vasc. Biol. 36(1), 122-33 (2016).

37. Anand P, Kunnumakkara AB, Sundaram C et al. Cancer is a preventable disease that requires major lifestyle changes. Pharm. Res. 25(9), 2097-2116 (2008).

38. Anand P, Kunnumakkara AB, Sundaram C et al. Tobacco smoke carcinogens, DNA damage and $\mathrm{p} 53$ mutations in smoking-associated cancers. Oncogene 21(48), 7435-7451 (2002).

39. Rothkamm K, Gunasekara K, Warda SA et al. Radiation-induced HPRT mutations resulting from misrejoined DNA double-strand breaks. Radiat. Res. 169(6), 639-648 (2008).

40. Wood RD. Nucleotide excision repair in mammalian cells. J. Biol. Chem. 272(38), 23465-23468 (1997).

41. Wang $\mathrm{H}$, Wang T, Guo $\mathrm{H}$ et al. Association analysis of ERCC5 gene polymorphisms with risk of breast cancer in Han women of northwest China. Breast Cancer 23(3), 479-485 (2016).

42. Gayarre J, Kamieniak MM, Cazorla-Jiménez A et al. The NER-related gene GTF2H5 predicts survival in high-grade serous ovarian cancer patients. J. Gynecol. Oncol. 27(1), e7 (2016).

43. Yu D, Zhang X, Liu J et al. Characterization of functional excision repair cross-complementation group 1 variants and their association with lung cancer risk and prognosis. Clin. Cancer Res. 14(9), 2878-2886 (2008).

44. Zhang R, Jia M, Xue H et al. Genetic variants in ERCC1 and XPC predict survival outcome of non-small-cell lung cancer patients treated with platinum-based therapy. Sci. Rep. 7(1), 10702 (2017).

45. Kim D, Fiske BP, Birsoy K et al. SHMT2 drives glioma cell survival in ischaemia but imposes a dependence on glycine clearance. Nature 520(7547), 363-367 (2015).

46. Hong Y, Huang X, An L et al. Overexpression of COPS3 promotes clear cell renal cell carcinoma progression via regulation of Phospho-AKT(Thr308), Cyclin D1 and Caspase-3. Exp. Cell Res. 365(2), 163-170 (2018).

47. Yan T, Tang G, Ren T et al. RNAi-mediated COPS3 gene silencing inhibits metastasis of osteogenic sarcoma cells. Cancer Gene Ther. 18(6), 450-456 (2011).

48. Pang J, Yan X, Cao $\mathrm{H}$ et al. Knockdown of COPS3 inhibits lung cancer tumor growth in nude mice by blocking cell cycle progression. $J$. Cancer 8(7), 1129-1136 (2017).

49. Xiao W, Wang J, Li H et al. Fibulin-1 is epigenetically down-regulated and related with bladder cancer recurrence. BMC Cancer 14(1), 677 (2014).

50. van Dartel M, Cornelissen PW, Redeker S et al. Amplification of $17 \mathrm{p} 11.2$ approximately p12, including PMP22, TOP3A and MAPK7 in high-grade osteosarcoma. Cancer Genet. Cytogenet. 139(2), 91-96 (2002).

51. Liu S, Chen Z. The functional role of PMP22 gene in the proliferation and invasion of osteosarcoma. Med. Sci. Monit. 21(7), 1976-1982 (2015).

52. Ranganathan P, Weaver KL, Capobianco AJ. Notch signaling in solid tumors: a little bit of everything but not all the time. Nat. Rev. Cancer. 11(5), 338-351 (2011).

53. Ntziachristos P, Lim JS, Sage J et al. From fly wings to targeted cancer therapies: a centennial for notch signaling. Cancer Cell 25(3), 318-334 (2014). 
54. Segade F, Suganuma N, Mychaleckyj JC et al. The intracellular form of human MAGP1 elicits a complex and specific transcriptional response. Int. J. Biochem. Cell Biol. 39(12), 2303-2313 (2007).

55. Werneck CC, Vicente CP, Weinberg JS et al. Mice lacking the extracellular matrix protein MAGP1 display delayed thrombotic occlusion following vessel injury. Blood 111(8), 4137-4144 (2008).

56. Craft CS, Pietka TA, Schappe T et al. The extracellular matrix protein MAGP1 supports thermogenesis and protects against obesity and diabetes through regulation of TGF- $\beta$. Diabetes 63(6), 1920-1932 (2014).

57. Mok SC, Bonome T, Vathipadiekal V et al. A gene signature predictive for outcome in advanced ovarian cancer identifies a survival factor: microfibril-associated glycoprotein 2. Cancer Cell 16(6), 521-532 (2009).

58. Marti P, Stein C, Blumer T et al. YAP promotes proliferation, chemoresistance and angiogenesis in human cholangiocarcinoma through TEAD transcription factors. Hepatology 62, 1497-1510 (2015).

59. Miyamoto A, Lau R, Hein PW et al. Microfibrillar proteins MAGP-1 and MAGP-2 induce Notch1 extracellular domain dissociation and receptor activation. J. Biol. Chem. 281(15), 10089-10097 (2006).

60. Koutelou E, Sato S, Tomomori-Sato C et al. Neuralized-like 1 (Neurl1) targeted to the plasma membrane by N-myristoylation regulates the Notch ligand Jagged1. J. Biol. Chem. 283(7), 3846-3853 (2008).

61. Teider N, Scott DK, Neiss A et al. Neuralized1 causes apoptosis and downregulates Notch target genes in medulloblastoma. Neuro. Oncol. 12(12), 1244-1256 (2010).

62. Wulfjohansson H, Johansson SL, Schlosser A et al. Localization of microfibrillar-associated protein 4 (MFAP4) in human tissues: clinical evaluation of serum MFAP4 and its association with various cardiovascular conditions. PLoS ONE 8(12), e82243 (2013). 
